\title{
Análisis de la Sensibilidad Paramétrica del Proceso de Producción de Ciclo-Trimetileno-Triamina
}

\author{
Juan C. Ojeda(1), Edison GilPavas(2), Izabela Dobrosz-Gómez(3), Miguel Á. Gómez ${ }^{(1)}$ \\ (1) Universidad Nacional de Colombia, Facultad de Ingeniería y Arquitectura, Departamento de Ingeniería \\ Química, Sede Manizales, PRISMA. Campus La Nubia, km 9, vía al Aeropuerto la Nubia, Apartado Aéreo \\ 127, Manizales-Colombia (e-mail: jcojedat@unal.edu.co; magomez@unal.edu.co) \\ (2) Departamento de Ingeniería de Procesos. Universidad EAFIT, GIPAB: Cr 49 \#7 sur 50, Medellín, \\ Colombia (e-mail: egil@eafit.edu.co) \\ (3) Universidad Nacional de Colombia, Facultad de Ciencias Exactas y Naturales, Departamento de Física \\ y Química, Sede Manizales, PRISMA. Campus La Nubia, km 9, vía al Aeropuerto la Nubia, Apartado Aéreo \\ 127, Manizales-Colombia (e-mail: idobrosz-gomez@unal.edu.co)
}

Recibido Dic. 16, 2013; Aceptado Mar. 14, 2014; Versión final recibida Mar. 28, 2014

\begin{abstract}
Resumen
Se realizó un análisis de sensibilidad paramétrica para la síntesis de ciclo-trimetileno-triamina, una reacción altamente exotérmica en cuya producción se deben garantizar y mantener condiciones estables. Utilizando el modelo de un reactor batch, se analizó la sensibilidad de la temperatura y la conversión con respecto a la temperatura inicial. Primero, se ajustó una expresión para la velocidad de reacción a partir de datos experimentales publicados. Luego, se usó un criterio simple basado en los parámetros de sensibilidad para determinar las condiciones críticas de operación mediante el análisis de las trayectorias de sensibilidad de la temperatura y conversión. Las condiciones críticas de reacciones en cadena corresponden con un número de Semenov $(\psi)$ igual a 0.684 , un parámetro de calor de reacción (B) igual a 15 y un número del tipo Arrhenius ( $\mathrm{Y}$ ) con un valor de 20.
\end{abstract}

Palabras clave: reacción de runaway, sensibilidad paramétrica, ciclo-trimetileno-triamina, criterios de operación estable

\section{Parametric Sensitivity Analysis for the Cyclo-Trimethylene- Triamine Production Process}

\begin{abstract}
Analyses of temperature, conversion, and their sensitivity with respect to the initial temperature were investigated by parametric sensitivity analysis using a dimensionless batch reactor model for the cyclotrimethylene-triamine synthesis. At first, an expression for its reaction rate was fitted from experimental data available in the literature. Then, a new simple sensitivity-based criterion was used to determine critical operating conditions analyzing temperature sensitivity trajectories. The critical condition of runaway reaction corresponds to a Semenov number $(\psi)$ equals to 0.684 , a heat of reaction parameter (B) equals to 15 and an Arrhenius-type number ( $\mathrm{Y}$ ) of 20.
\end{abstract}

Keywords: runaway reaction, parametric sensitivity, cyclo-trimethylene-triamine, stable reaction criteria 


\section{INTRODUCCIÓN}

La producción de ciclo-trimetileno-triamina, un explosivo también conocido como RDX, Hexogeno, T4 o ciclonita, mediante la reacción en fase líquida de hexamina con ácido nítrico, ha sido ampliamente usada desde la segunda guerra mundial (1939) (Luo et al., 2002). Esta reacción es altamente exotérmica (-90938 $\mathrm{kJ} / \mathrm{mol}$ de hexamina) y muy sensible a la variación de la temperatura. De hecho, si se considera este proceso reactivo en un reactor batch, cuando la tasa de calor total generado excede la tasa de calor removido por un refrigerante, el sistema puede acumular fácilmente energía y elevar rápidamente su temperatura. Así, cuando la temperatura en el reactor alcanza el punto crítico, el sistema reactivo se puede encontrar en una situación fuera de control (runaway) o incluso explosión. Este fenómeno corresponde a la alta sensibilidad térmica del proceso reactivo que eventualmente puede conducir a una condición de runaway térmico (Willey et al., 2010).

En años recientes, la operación térmica segura de reactores ha incrementado su interés con el fin de evitar los accidentes del tipo runaway los cuales pueden generar muerte, daños a instalaciones y contaminación ambiental (CSB, 2013). Son muchas las metodologías propuestas para el análisis de las condiciones de runaway. En un primer intento, Semenov (1928) definió que las condiciones críticas dependen de la forma en que crece la curva de temperatura. Luego, Kamenetskii (1939) asumió que el enfriamiento en un reactor químico está controlado por la conducción térmica en el seno de la mezcla reactiva. Estos métodos predicen las condiciones de runaway térmico mediante un análisis de estabilidad lineal del balance de energía. El concepto de sensibilidad paramétrica fue propuesto inicialmente por Bilous y Amundson (1956). Ellos establecieron que un reactor químico opera en la región de sensibilidad paramétrica cuando pequeños cambios en los valores de uno o más parámetros de entrada al reactor generan grandes cambios (v.g., runaway) en las variables de salida del mismo. De esta forma se desarrollaron criterios basados en las características geométricas de los perfiles de temperatura y/o conversión (Thomas y Bowes (1961); Adler y Enig (1964); van Walsenaere y Froment (1970)).

En general, se podría decir que en las últimas dos décadas, la estimación de los límites entre las regiones con condiciones de operación seguras y de runaway se ha enfrentado siguiendo tres principales metodologías: el método de sensibilidad paramétrica (Morbidelli y Varma, 1988; Varma et al., 1999); el método de la divergencia basado en la teoría del caos (Strozzi, y Zaldivar, 1994) y el método de la extensión de trayectorias (Bosch et al., 2004). Así, Morbidelli y Varma (1988) desarrollaron un criterio de sensibilidad paramétrica generalizado, en el cual la región de sensibilidad paramétrica se identifica como aquella donde la sensibilidad objetivo normalizada del máximo de temperatura, con respecto a cualquier parámetro de entrada del modelo, alcanza un máximo.

A pesar de la experiencia acumulada, se han reportado numerosos incidentes y calamidades relacionados con el proceso de producción de ciclo-trimetileno-triamina (Kletz, 2009). De hecho, hasta donde conocemos, el único trabajo que se encuentra en la literatura relacionado con la evaluación de las condiciones críticas de operación y los criterios de estabilidad de este sistema (en un reactor batch) fue el desarrollado por Luo et al., (2002) basado en el criterio de Kamenetskii (1939). Su método de análisis incluyó parametrizar los balances de materia y energía en variables adimensionales, determinar los puntos calientes presentes (máxima elevación de temperatura) a ciertas condiciones de operación, y definió como criterio de sensibilidad los valores de los parámetros y el tiempo en el que $\mathrm{d} \theta / \mathrm{d} T \rightarrow \propto$.

Poco después, Lu et al., (2005) extrapolaron esta metodología a un reactor CSTR. En este caso se realizó una comparación entre el cambio de los perfiles del calor generado por la reacción y del calor removido por un medio refrigerante con respecto a las variables de operación; se determinan los múltiples estados de operación (estables e inestables) y por último se define las regiones críticas en las cuales existe sensibilidad térmica del sistema. Más recientemente, Ball y Gray (2013) analizaron la dinámica no lineal y bifurcaciones que presenta este sistema a ciertas condiciones, mostrando la sensibilidad de la temperatura del reactor respecto a la temperatura del fluido de servicio, para diferentes valores de caudal, determinando las condiciones en las cuales el sistema es completamente inestable.

El objetivo de este trabajo es obtener un nuevo y simple criterio de sensibilidad para determinar las condiciones críticas de operación de un reactor batch para la producción de ciclo-trimetileno-triamina utilizando el modelo generalizado de Morbidelli y Varma (1988). Este incluye el análisis de los perfiles de temperatura y concentración así como de sensibilidad de la temperatura en función de las variables de operación. Así, se analizó el efecto del número de Semenov, el parámetro del calor de reacción y un número adimensional del tipo Arrhenius en la temperatura del reactor y su sensibilidad. Para el modelamiento, se ajustó una nueva expresión cinética a partir de los datos experimentales reportados por Dai et al., (1996). 


\section{EVALUACIÓN DE LOS PARÁMETROS CINÉTICOS}

La reacción química de hexamina $\left(\mathrm{C}_{6} \mathrm{H}_{12} \mathrm{~N}_{4}\right)$ y ácido nítrico $\left(\mathrm{HNO}_{3}\right)$ para producir ciclo-trimetileno-triamina $\left(\mathrm{C}_{3} \mathrm{H}_{6} \mathrm{~N}_{6} \mathrm{O}_{6}\right)$ está dada por la ecuación (1).

$$
\mathrm{C}_{6} \mathrm{H}_{12} \mathrm{~N}_{4}+10 \mathrm{HNO}_{3} \rightarrow \mathrm{C}_{3} \mathrm{H}_{6} \mathrm{~N}_{6} \mathrm{O}_{6}+3 \mathrm{CH}_{2}\left(\mathrm{ONO}_{2}\right)_{2}+\mathrm{NH}_{4} \mathrm{NO}_{3}+3 \mathrm{H}_{2} \mathrm{O}
$$

Existe además la posibilidad de una reacción secundaria con los mismos reactantes:

$$
\mathrm{C}_{6} \mathrm{H}_{12} \mathrm{~N}_{4}+16 \mathrm{HNO}_{3} \rightarrow 6 \mathrm{CH}_{2}\left(\mathrm{ONO}_{2}\right)_{2}+4 \mathrm{NH}_{4} \mathrm{NO}_{3}
$$

Dai et al., (1996) realizaron experimentos en un reactor batch con masas de alimentación de ácido nítrico, $\mathrm{W}_{\mathrm{HNO}}$, y de hexamina, $\mathrm{W}_{\mathrm{A}}$, iguales a 110 y $10 \mathrm{~g}$, respectivamente. Para valores fijos de tiempo de reacción y temperatura, midieron la masa de producción de ciclo-trimetileno-triamina, $W_{\mathrm{RDX}}$. De esta forma, la tasa de rendimiento de ciclo-trimetileno-triamina, $Y(\%)$, y la conversión de hexamina, $x_{A}$, pueden estimarse a partir de los datos experimentales (Tabla 1).

Tabla 1: Producción de ciclo-trimetileno-triamina a diferentes tiempos de reacción y temperatura (Dai et al., 1996)

\begin{tabular}{|c|c|c|c|c|}
\hline $\mathrm{T},{ }^{\circ} \mathrm{C}$ & $\mathrm{T}, \min$ & $\mathrm{W}_{\mathrm{RDX}}$ & $\mathrm{Y}(\%)$ & $\mathrm{x}_{\mathrm{A}}$ \\
\hline-25 & 600 & 13.20 & 83 & 0.83 \\
\hline-10 & 360 & 13.10 & 83 & 0.83 \\
\hline 0 & 120 & 13.00 & 82 & 0.82 \\
\hline 10 & 45 & 12.95 & 82 & 0.82 \\
\hline 20 & 15 & 12.80 & 81 & 0.81 \\
\hline 30 & 10 & 12.75 & 80 & 0.80 \\
\hline 35 & 19 & 12.75 & 80 & 0.80 \\
\hline 40 & 5 & 12.50 & 79 & 0.79 \\
\hline 50 & 5 & 11.50 & 73 & 0.73 \\
\hline 60 & 5 & 9.97 & 63 & 0.63 \\
\hline
\end{tabular}

Considerando la estequiometria de la reacción, la relación másica estequiométrica entre el ácido nítrico y la hexamina, $\mathrm{W}_{\mathrm{HNO}} / \mathrm{W}_{\mathrm{A}}$, es igual a 2.84. En los experimentos, las cantidades utilizadas dan una relación másica de 11. Por lo tanto, se puede asumir que la concentración de ácido nítrico en los experimentos sólo sufrirá una variación menor (se mantendrá constante). En consecuencia, la velocidad de reacción de hexamina en el sistema de ácido nítrico se puede expresar como:

$-\mathrm{r}_{\mathrm{A}}=\mathrm{kC}_{\mathrm{HNO}_{3}} \mathrm{C}_{\mathrm{A}}^{\mathrm{n}}$

donde $\mathrm{n}, \mathrm{k}=\mathrm{k}_{\mathrm{O}} \mathrm{e}^{\mathrm{E} / \mathrm{RT}}$ y $\mathrm{C}_{\mathrm{HNO}}$ son el orden de reacción, la constante de velocidad y la concentración inicial de ácido nítrico, respectivamente. Sustituyendo la ecuación (3) en el balance molar para un reactor batch y realizando un ajuste no lineal de los datos mediante el método Simplex de Nelder-Mead (disponible en la función fminsearch de $M a t L a b^{\circledR}$ ) se pueden obtener los valores de los parámetros cinéticos (factor de frecuencia $\left(\mathrm{k}_{0}\right)$ y la energía de activación (E)) de la ecuación (3). Estos se presentan en la Tabla 2. El método Simplex de Nelder-Mead es un algoritmo de optimización, el cual minimiza una función objetivo multivariable. En este caso la función objetivo corresponde a la expresión (4). Se obtiene un valor por cada dato experimental.

Tabla 2: Parámetros cinéticos calculados a partir de los datos experimentales reportados por Dai et al., (1996)

\begin{tabular}{|c|c|}
\hline Orden de la reacción & $\mathrm{n}=1.28$ \\
\hline Factor de frecuencia & $\mathrm{k}_{0}=7.042 \times 10^{5}[\mathrm{~L} / \mathrm{mol}]^{1.28} \mathrm{~min}^{-1}$ \\
\hline Energía de activación & $\mathrm{E}=46.138 \mathrm{~kJ} / \mathrm{mol}$ \\
\hline
\end{tabular}


Para corroborar la bondad del ajuste de los parámetros cinéticos, se linealizó el balance molar del reactor batch y se calculó el coeficiente de correlación lineal $\left(r^{2}\right)$. Así, sustituyendo la ecuación (3) en el balance molar del reactor batch, expresándolo en términos de la conversión e integrando analíticamente, se obtiene

$$
\frac{t}{\left(1-x_{A}\right)^{1-n}-1}=\frac{C_{A 0}^{1-n} e^{E / R T}}{C_{\mathrm{HNO}_{3}}(n-1) k_{0}}
$$

La ecuación (4) se linealiza, tomando algoritmo natural a ambos lados

$$
\ln \left[\frac{\mathrm{t}}{\left(1-\mathrm{x}_{\mathrm{A}}\right)^{1-n}-1}\right]=\ln \left[\frac{\mathrm{C}_{\mathrm{A} 0}^{1-n}}{\mathrm{k}_{0}(\mathrm{n}-1) \mathrm{C}_{\mathrm{HNO}_{3}}}\right]+\frac{\mathrm{E}}{\mathrm{RT}}
$$

La expresión (5) tiene la forma de una línea recta, donde E/R corresponde a la pendiente y $\ln \left[t /\left(1-x_{A}\right)^{1-n}-1\right]$ corresponde al corte con el eje vertical. En la Figura 1 se muestra el ajuste lineal de los datos experimentales. El procedimiento de ajuste (minimización) dio como resultado un orden de reacción $\mathrm{n}=1.28$, con un coeficiente de correlación de 0.9386 .

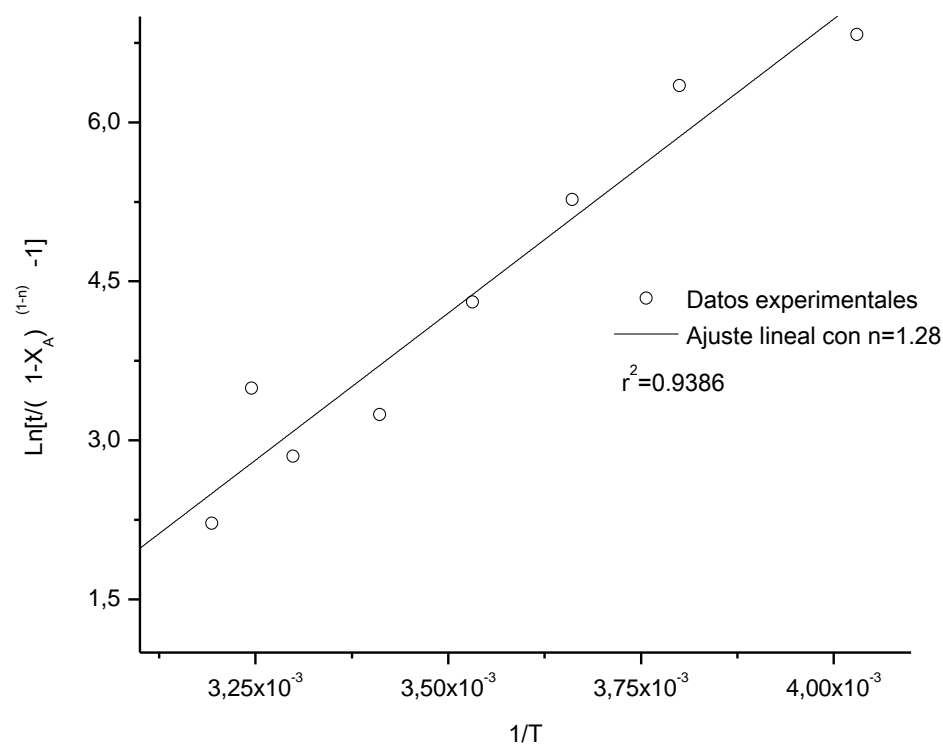

Fig. 1: Evaluación de los parámetros cinéticos del sistema de reacción batch hexamina-ácido nítrico para la producción de ciclo-trimetileno-triamina

\section{COEFICIENTE DE SENSIBILIDAD}

Considérese que el reactor batch se opera a condiciones homogéneas y bajo condiciones isoperibólicas (v.g., la temperatura del fluido de servicio en la chaqueta del reactor se mantiene constante). Es posible demostrar que el balance de molar y de energía para ese reactor son de la siguiente forma:

$$
\begin{aligned}
& \frac{d x_{A}}{d t}=k_{0} \exp \left(-\frac{E}{R T}\right) C_{H_{N O}} C_{A 0}^{n-1}\left(1-x_{A}\right)^{n} \\
& \frac{d T}{d t}=\frac{q_{g} k_{0} \exp \left(-\frac{E}{R T}\right) C_{H N O_{3}} C_{A 0}^{n-1}\left(1-x_{A}\right)^{n}}{\rho C_{p m}}-\frac{h S}{\rho V C_{p m}}\left(T-T_{a}\right)
\end{aligned}
$$


Donde $q_{g}$ es el calor de generación, $\rho$ es la densidad de la mezcla, $\mathrm{C}_{\mathrm{pm}}$ es la capacidad calorífica de la mezcla, $h$ es el coeficiente de transferencia de calor, $\mathrm{S}$ es el área de transferencia de calor, $\mathrm{V}$ es el volumen del reactor y $\mathrm{T}_{\mathrm{a}}$ es la temperatura del refrigerante. Los balances están sujetos a las siguientes condiciones iniciales:

$\mathrm{t}=0, \quad \mathrm{x}_{\mathrm{A}}=0, \quad \mathrm{~T}=\mathrm{T}_{0}$

Las ecuaciones (7) y (8) pueden ser rescritas en forma adimensional como:

$$
\begin{aligned}
& \frac{d x_{A}}{d t}=\exp \left(\frac{\theta}{1+\theta / Y}\right)\left(1-x_{A}\right)^{n} \\
& \frac{d \theta}{d \tau}=B \exp \left(\frac{\theta}{1+\theta / Y}\right)\left(1-x_{A}\right)^{n}-\frac{B}{\psi}\left(\theta-\theta_{a}\right)
\end{aligned}
$$

y las condiciones iniciales quedan como:

$\mathrm{T}=0, \quad \mathrm{x}_{\mathrm{A}}=0, \quad \theta=0$

Las variables adimensionales están definidas por:

Temperatura adimensional

$\theta=\frac{T-T_{0}}{T_{0}} Y$

Conversión de hexamina

$$
\begin{aligned}
& x_{A}=\frac{C_{A 0}-C_{A}}{C_{A 0}} \\
& T=k\left(T_{0}\right) C_{\mathrm{HNO}_{3}} C_{A 0}^{n-1} t
\end{aligned}
$$

y los parámetros adimensionales relacionados de la siguiente manera:

Incremento de la temperatura adimensional adiabática

$$
\mathrm{B}=\frac{\mathrm{q}_{\mathrm{g}} \mathrm{C}_{\mathrm{A} 0}}{\rho \mathrm{C}_{\mathrm{pm}} \mathrm{T}_{0}} \mathrm{Y}=\frac{\text { Calor Generado por la Reacción }}{\text { Calor Sensible de la Mezcla Reactiva }}
$$

Energía de activación adimensional

$$
\mathrm{Y}=\frac{\mathrm{E}}{\mathrm{RT_{0 }}}=\frac{\text { Energía de Activación }}{\text { Temperatura Inicial del Sistema }}
$$

Número de Semenov

$\Psi=\frac{\mathrm{q}_{\mathrm{g}} \mathrm{k}\left(\mathrm{T}_{0}\right) \mathrm{C}_{\mathrm{HNO}_{3}} \mathrm{C}_{\mathrm{A} 0}^{\mathrm{n}}}{\mathrm{hST}_{0}} \mathrm{~V}=\frac{\text { Calor Generado por la Reacción }}{\text { Calor Removido por el Fluido de Servicio }}$

donde $\psi$ representa la relación entre el potencial del calor generado y el potencial del calor removido por el fluido de servicio.

Para determinar los coeficientes de sensibilidad se tienen en cuenta los balances molar y de energía mediante una ecuación diferencial general (18) que describe el comportamiento dinámico, tal y como lo sugieren Morbidelli y Varma (1988): 


$$
\frac{d y}{d t}=f(y, \varphi, T)
$$

\section{Con condición inicial}

$$
\mathrm{y}(0)=\mathrm{y}_{0}
$$

Se asume que la función f es continua y diferenciable en todos sus argumentos. Una posible solución a la ecuación puede ser:

$$
y=y(T, \varphi)
$$

Si se genera una pequeña perturbación al j-ésimo parámetro de entrada, de $\varphi_{\mathrm{j}}$ a $\varphi_{\mathrm{j}}+\Delta \varphi_{\mathrm{j}}$, la variable dependiente se modificará según la expresión $\mathrm{y}=\mathrm{y}\left(\mathbf{T}, \varphi_{\mathrm{j}}+\Delta \varphi_{\mathrm{j}}\right)$. Así, se define el coeficiente de sensibilidad de primer orden $\mathrm{S}\left(\mathrm{y} ; \varphi_{\mathrm{j}}\right)$ como:

$$
S\left(y ; \varphi_{j}\right)=\frac{\partial y}{\partial \varphi_{j}}=\lim _{\Delta \varphi_{j} \rightarrow 0} \frac{y\left(\varphi_{j}+\Delta \varphi_{j}\right)-y\left(\varphi_{j}\right)}{\Delta \varphi_{j}}
$$

El valor del coeficiente de sensibilidad puede ser positivo o negativo. Cuando éste es positivo, un aumento en los parámetros de entrada conduce a un incremento en la variable dependiente correspondiente; cuando es negativo sucede lo contrario. Existen tres métodos para evaluar los coeficientes de sensibilidad: el método diferencial directo (Rabitz et al., 1983), el método de las diferencias finitas (Kramer et al., 1984) y el método de la función de Green (Hwang et al., 1978). Para el caso bajo estudio se aplicará el método diferencial directo considerando su fácil implementación computacional (v.g., en el software MatLab ${ }^{\circledR}$ ). Así, diferenciando ambos lados de la ecuación (19) con respecto a $\varphi_{\mathrm{j}}$, se obtiene:

$$
\frac{d\left(\partial y / \partial \varphi_{j}\right)}{d t}=\frac{d S\left(y ; \varphi_{j}\right)}{d t}=\frac{\partial f}{\partial y} \frac{\partial y}{\partial \varphi_{j}}+\frac{\partial f}{\partial \varphi_{j}}=\frac{\partial f}{\partial y} S\left(y ; \varphi_{j}\right)+\frac{\partial f}{\partial \varphi_{j}}
$$

La variable dependiente y incluye la conversión y la temperatura del reactante. El parámetro de entrada $\varphi_{\mathrm{j}}$ puede ser la temperatura inicial o el flujo del refrigerante, etc. Si los coeficientes de sensibilidad de la conversión y la temperatura son denotados con respecto a la temperatura inicial, entonces las ecuaciones de sensibilidad pueden ser expresadas de la siguiente forma:

$$
\begin{aligned}
& \frac{d S\left(x_{A} ; \theta_{0}\right)}{d T}=\frac{d x_{A}}{d \tau}\left(\frac{S\left(\theta ; \theta_{0}\right)}{(1+\theta / Y)^{2}}-\frac{n S\left(x_{A} ; \theta_{0}\right)}{1-x_{A}}\right) \\
& \frac{d S\left(\theta ; \theta_{0}\right)}{d \tau}=B \frac{d S\left(x_{A} ; \theta_{0}\right)}{d \tau}-\frac{B}{\psi} S\left(\theta ; \theta_{0}\right)
\end{aligned}
$$

Con condiciones iniciales:

$$
T=0, \quad S\left(x_{A} ; \theta_{0}\right)=0, \quad S\left(\theta ; \theta_{0}\right)=1
$$

Para analizar la variación de la temperatura del reactor (variable dependiente $\theta$ ) y su sensibilidad, $S\left(\theta ; \theta_{0}\right)$, en función del tiempo ( $\mathrm{T}$ ), se resuelven las ecuaciones (9) y (10), balance molar y de energía, y las ecuaciones (23) y (24), de sensibilidad paramétrica, junto con las condiciones iniciales (11) y (25). Los resultados obtenidos se presentan a continuación.

\section{ANÁLISIS DEL COMPORTAMIENTO DE LA TEMPERATURA Y SENSIBILIDAD}

La Figura 2 describe el perfil de temperatura adimensional en el reactor y su sensibilidad con la temperatura inicial en función del tiempo para cinco valores diferentes del número de Semenov $(\psi)$. Se puede observar que para curvas con valores del número de Semenov menor que 0.684 , la temperatura aumenta lentamente 
con el tiempo y la correspondiente sensibilidad disminuye gradualmente. Esto significa que a esas condiciones la temperatura del reactor no cambia considerablemente o que, en otras palabras, no muestra ninguna sensibilidad. En la Figura 2 (derecha) se puede observar que el valor crítico del número de Semenov es 0.684 (el límite de la condición de estabilidad determinado por el máximo valor de la función de sensibilidad de la temperatura que es igual a 1 : v.g., $\max \left(\mathrm{S}\left(\theta ; \theta_{0}\right)\right)=1$ excepto en $\mathrm{T}=0$, identificada por la línea horizontal de la Figura 2-derecha); por encima de ese valor, la temperatura del reactor aumenta rápidamente y el correspondiente valor de sensibilidad primero disminuye y luego aumenta pasando tangencialmente por el punto $S\left(\theta ; \theta_{0}\right)=1$ en $\mathrm{T}=0.15$. En variables no adimensionales, este valor crítico infiere que el calor generado por la reacción no puede superar 0.684 veces el calor removido por el fluido de servicio a través de la pared del reactor. Si se llegará a superar esta relación, el reactor incrementaría su temperatura descontroladamente hasta alcanzar un punto caliente con consecuencias catastróficas para el proceso (v.g., la destrucción o explosión del reactor).
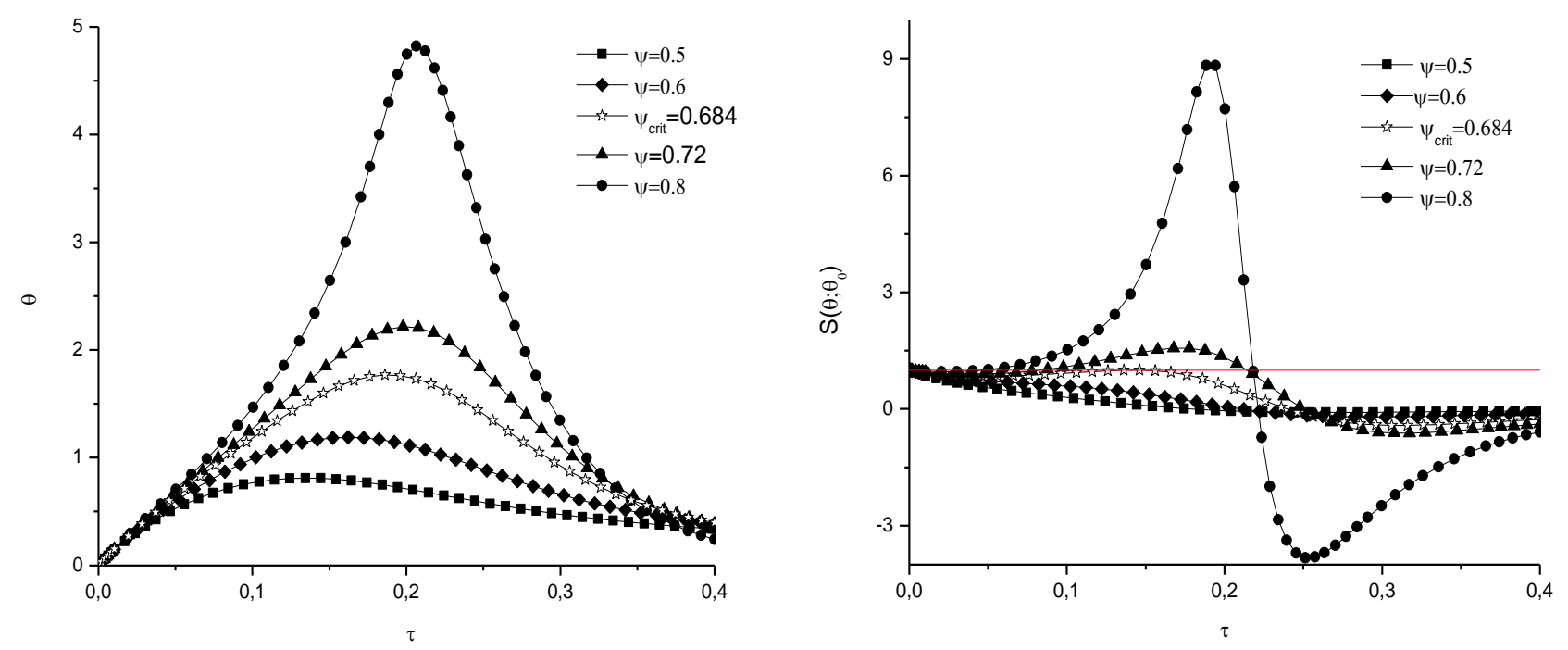

Fig. 2: Perfil de temperatura adimensional (izquierda) y de sensibilidad la temperatura adimensional (derecha) a diferentes valores de Semenov.

Parámetros: $\mathrm{B}=15 ; \mathrm{Y}=20 ; \theta_{\mathrm{a}}=0$

De forma análoga a los resultados presentados en la Figura 2, usando el número de Semenov como parámetro, en la Figura 3 se presenta el perfil de conversión de hexamina en el reactor y su sensibilidad con la temperatura inicial en función del tiempo. Se observa que a valores de $\psi>0.684$ los perfiles de conversión empiezan a crecer más rápidamente. A valores de $\psi>0.8$, la conversión máxima se alcanza en un valor tiempo adimensional de ca. $\mathbf{T}=0.22$, asíntotizándose en $\mathrm{X}_{\mathrm{A}}=0.92$ a valores de tiempo adimensional superiores disminuyendo su sensibilidad paramétrica. En este caso, como la temperatura aumenta rápidamente con el tiempo (para $\psi>0.684$ ), la velocidad de reacción también incrementa (dependencia de la ecuación de Arrhenius) y por lo tanto la conversión alcanza incluso valores cercanos a la unidad.

La variación de la temperatura del reactor y su sensibilidad en función del aumento adimensional de la temperatura adiabática (B) se compara en la Figura 4. Estas presentan un comportamiento similar al observado en función del número de Semenov. El parámetro B tiene un valor crítico de 15 (Figura 4 izquierda). Cuando este límite es superado, la reacción es inestable y se encuentra fuera de control, debido a la excesiva elevación de la temperatura producto del calor generado por la reacción. Esto puede ocurrir cuando la concentración del reactante es considerable o cuando la temperatura de alimento no es lo suficientemente baja. En la Figura 4 (derecha) se muestra la sensibilidad que tiene la temperatura adimensional con respecto a la temperatura inicial a diferentes valores de $\mathrm{B}$. Se puede observar con más claridad que a valores mayores de $B_{\text {crit, }}$ el sistema es muy sensible y tiende a que la temperatura incremente rápidamente en un corto tiempo (la energía liberada es mucho mayor que la removida). 

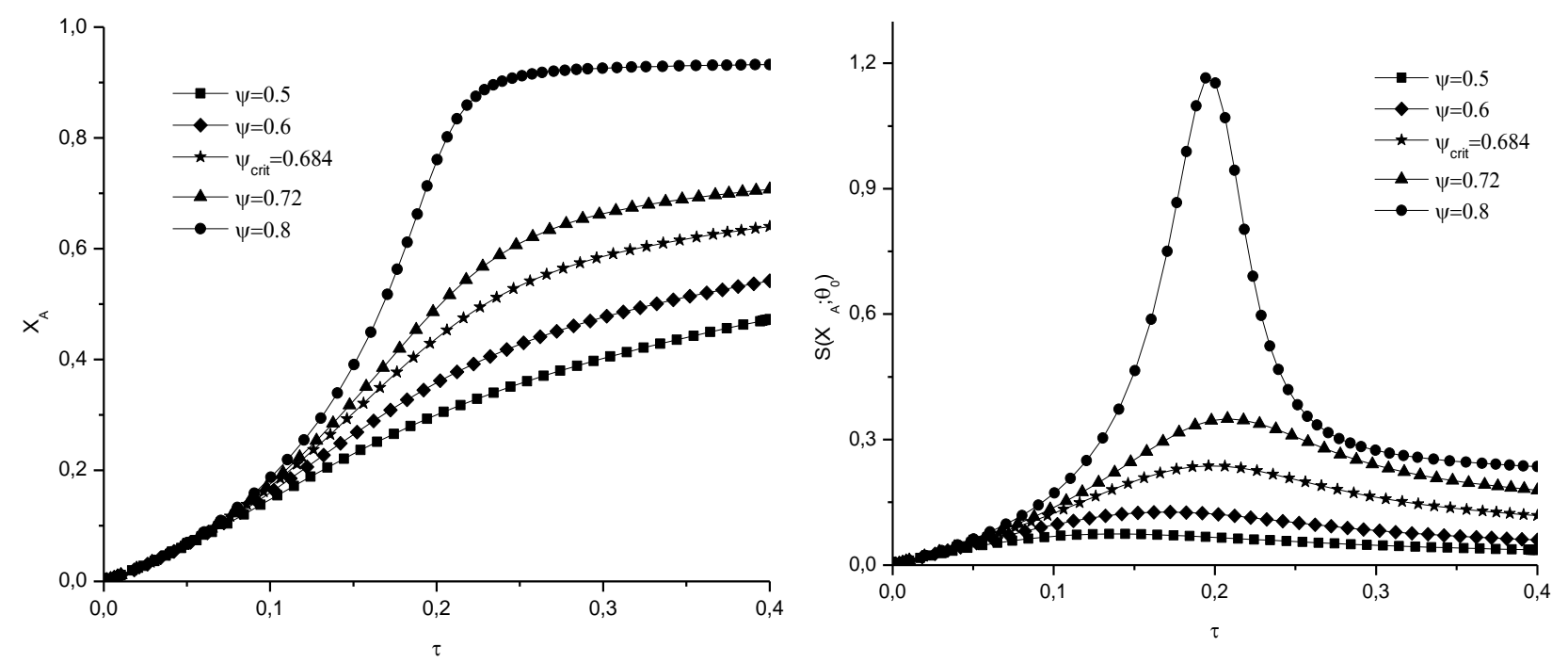

Fig. 3: Perfil de conversión (izquierda) y de sensibilidad de la conversión con la temperatura inicial (derecha) a diferentes valores de Semenov. Parámetros: $B=15 ; y=20 ; \theta_{a}=0$
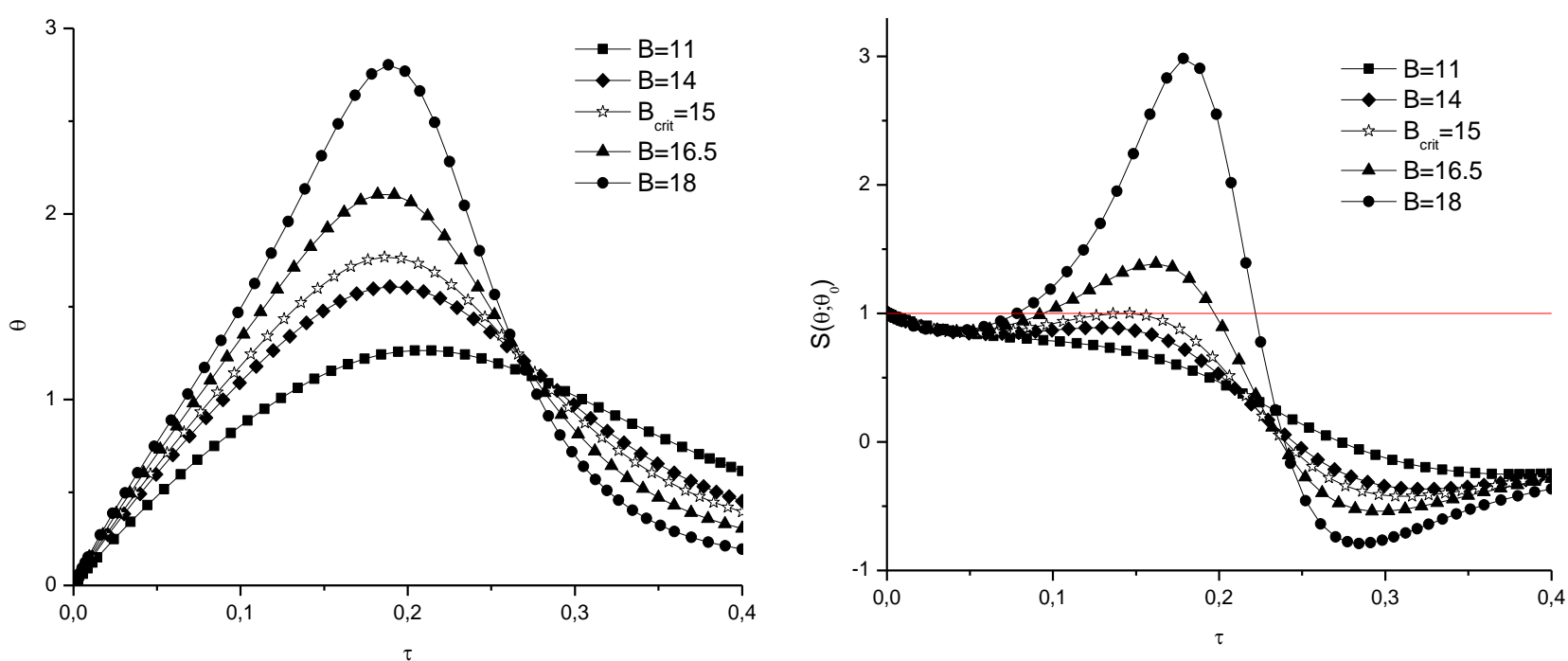

Fig. 4: Perfil de temperaturas adimensionales (izquierda) y sensibilidad de la temperatura adimensional con la temperatura inicial (derecha) a diferentes valores de B. Parámetros: $\psi=0.684 ; \gamma=20 ; \theta_{a}=0$

El parámetro Y, que representa la energía de activación adimensional, tiene un valor crítico de 20 (Figura 5 izquierda). Por encima de este valor el sistema presenta un tránsito a la región fuera de control, esto debido a la drástica elevación de temperatura producto de la energía liberada cuando se lleva a cabo la reacción. De hecho, para un $\mathrm{y}=20$ la temperatura es muy sensible y se puede incrementar fácilmente con una pequeña variación en las condiciones iniciales de operación (temperatura de alimentación). En el valor crítico de $\mathrm{Y}$, el coeficiente de sensibilidad alcanza un máximo que corresponde a la unidad en un tiempo de $\mathrm{T}=0.15$ (Figura 5 derecha). Cabe resaltar que los valores de los parámetros del incremento de la temperatura adiabática y el número de Semenov se encuentran íntimamente relacionados con el valor de la energía de activación. Son directamente proporcionales.

Los resultados de un cálculo de las condiciones críticas de runaway realizado a condiciones típicas de operación para el sistema reactivo bajo estudio, utilizando el criterio de Kamenetskii, fue reportado por Luo et al., (2002). Comparando sus resultados con los obtenidos en este estudio, se observa que se coincide con el valor crítico de $y=20$. A esa condición, el valor de la temperatura adimensional reportada por Luo et al., (2002) es de 2.17 mientras que el predicho por modelo utilizado en este estudio es de 1.72. La 
diferencia entre estos valores puede explicarse del hecho que la simulación realizada en esta investigación involucra simultáneamente los valores críticos de los otros parámetros (v.g., B y $\psi$ ). Así, puede considerarse que el nuevo criterio propuesto aquí puede proporcionar predicciones más conservadoras de los límites seguros de desempeño del reactor batch.
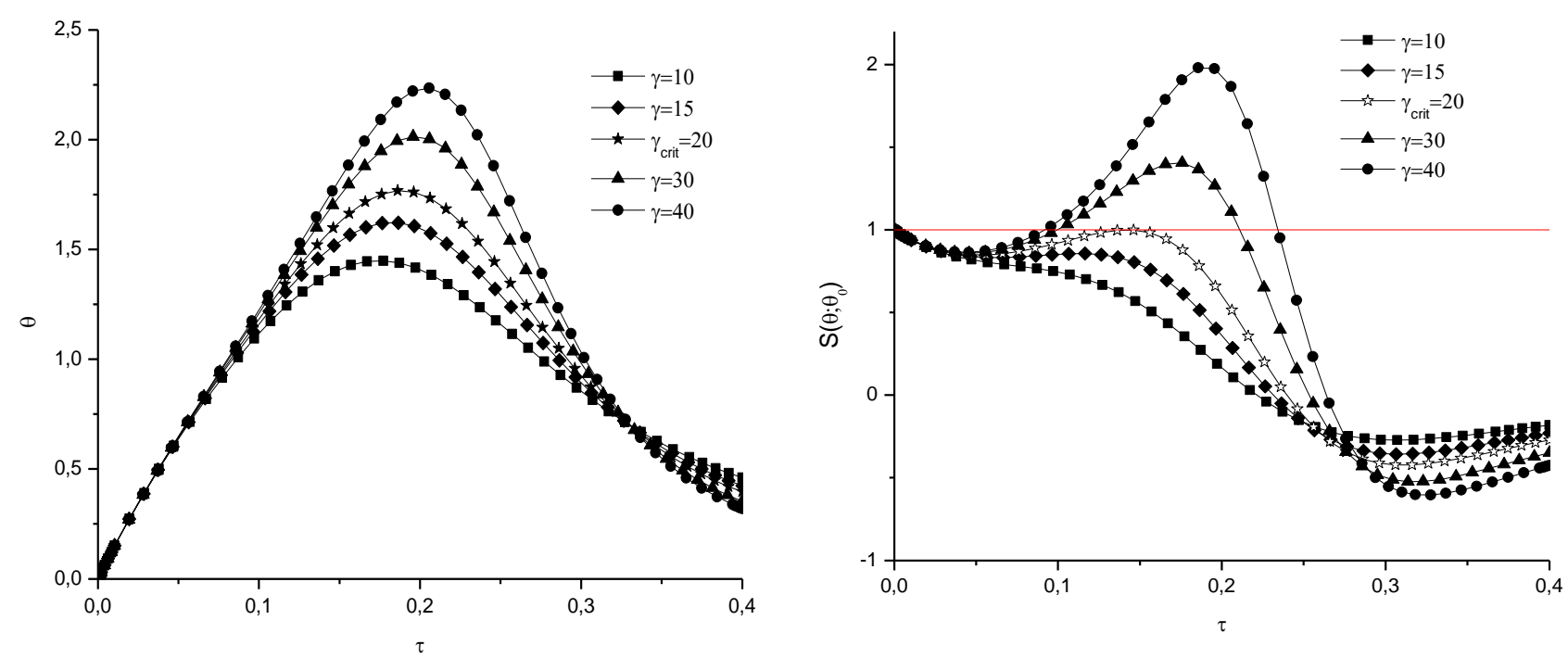

Fig. 5: Perfil de temperaturas adimensionales (izquierda) y sensibilidad de la temperatura adimensional con la temperatura inicial (derecha) a diferentes valores del parámetro $\gamma$. Parámetros: $\psi=0.684 ; B=15 ; \theta_{a}=0$

\section{CONCLUSIONES}

En este trabajo se implementó numéricamente un criterio simple para evaluar la sensibilidad paramétrica de la temperatura de reacción, la conversión y sus sensibilidades dinámicas para el proceso de producción de ciclo-trimetileno-triamina. Los resultados demuestran que el proceso batch exhibe alta sensibilidad en el espacio paramétrico. Así, pequeñas variaciones en los parámetros de entrada generan grandes cambios en las variables de salida. Los valores críticos de los parámetros del sistema son: La relación entre la energía generada por la reacción y la energía removida por el fluido de servicio es igual a $\psi=0.684$, la relación entre la energía generada por la reacción y la energía sensible (energía absorbida) de la mezcla reactiva es igual a $B=15$ y la energía de activación adimensional tiene un valor de $\mathrm{y}=20$.

\section{AGRADECIMIENTOS}

Los autores agradecen apoyo económico de la DIMA (Programa para la financiación de Semilleros de Investigación en Emprendimiento en la Universidad Nacional de Colombia Sede Manizales - 2012- código 17037) y de COLCIENCIAS (Programa Jóvenes Investigadores e Innovadores Convocatoria 566 de 2012 Convenio Especial de Cooperación No. 0729 de 2012).

\section{REFERENCIAS}

Adler, J. y J.W. Enig, The Critical Conditions in Thermal Explosion Theory with Reactant Consumption, Combustion and Flame: 8, 97-103 (1964).

Ball, R. y B. F. Gray, Thermal instability and runaway criteria: The dangers of disregarding dynamics, Process Safety and Environmental Protection, 91, 221-226 (2013).

Bilous, O. y N. R. Amundson, Chemical Reactor Stability, AIChE Journal, 2, 117-126 (1956). 
Bosch, J., y otros cuatro autores, A comparative analysis between temperature and pressure measurements for early detection of runaway initiation, Journal of Loss Prevention in the Process Industries, 17(6), 389 395 (2004).

Chemical Safety Board, CSB, U.S.A. http://www.csb.gov/. Acceso: 15 Marzo (2013).

Dai, R. Z., S. E. Yen, y. K. Chen, The Production Technology and Design of Explosives, Weapon Industrial Pub. Co. (1996).

Hwang, J. T. y otros tres autores, The Green's function method of sensitivity analysis in chemical kinetics, Journal of Chemistry and Physics, 69, 5180 - 5191 (1978).

Jiang, J. y otros cuatro autores, Investigation on Thermal Runaway in Batch Reactors by Parametric Sensitivity Analysis, Chemical Engineering \& Technology, 34( 9), 1521-1528 (2011).

Kamenetskii, F. Diffusion and Heat Transfer in Chemical Kinetic, $2^{\mathrm{a}}$ edición, Plenum Press, New York, U.S.A. (1969).

Kletz, T. What Went Wrong?: Case Histories of Process Plant Disasters and How They Could Have Been Avoided, Butterworth-Heinemann (2009).

Kramer, M. A. y otros tres autores, Sensitivity analysis in chemical kinetics: recent developments and computational comparisons, International Journal of Chemical Kinetics, 16, 559 - 578 (1984).

Lu, K. T. y otros tres autores, Critical runaway conditions and stability criterion of RDX manufacture in continuous stirred tank reactor, Journal of Loss Prevention in the Process Industries, 18, 1-11 (2005).

Luo, K. M. y otros tres autores, Evaluations of kinetic parameters and critical runaway conditions in the reaction system of hexamine-nitric acid to produce $R D X$ in a non-isothermal batch reactor, Journal of Loss Prevention in the Process Industries, 15, 119-127 (2002).

Morbidelli, M., y A. Varma, A generalized criterion for parametric sensitivity: application to thermal explosion theory, Chemical Engineering Science, 43, 91 - 102 (1988).

Rabitz, H.; M. Kramer y D. Dacol, Sensitivity analysis in chemical kinetics, Annual Review of Physical Chemistry, 34, 419 - 461 (1983).

Semenov N. N., Zur Theorie des Verbrennungsprozesses, Zeitschrift für Physik, 48(7-8), 571-582 (1928).

Strozzi, F. y J. M. Zaldivar, A general method for assessing the thermal stability of batch chemical reactors by sensitivity calculation based on Lyapunov exponents, Chemi. Engin. Science, 49, 2681-2688 (1994).

Thomas, P. H. y P. C. Bowes, Some aspects of the ignition and self-heating of solid cellulosic materials, Journal of Applied Physics., 12, 222 (1961).

Varma, A., M. Morbidelli, y H. Wu, Parametric Sensitivity in Chemical Systems, Cambridge Series in Chemical Engineering, Cambridge University Press. (1999).

Van Welsenaere, R. J. y G. F. Froment, Parametric Sensitivity and Runaway in Fixed Bed Catalytic Reactors, Chemical Engineering Science, 25, 1503-1516 (1970).

Willey, R. J., H. S. Fogler, y M. B. Cutlip, The integration of Process Safety into a Chemical Reaction Engineering Course: Kinetic Modeling of the T2 Incident, AIChE Journal, 30(1), 39 - 44 (2010). 